\title{
Heat in optical tweezers
}

B. del Rosal, P. Haro-González, W. T. Ramsay, L. M. Maestro, K. Santacruz-Gómez, et al.

B. del Rosal, P. Haro-González, W. T. Ramsay, L. M. Maestro, K. SantacruzGómez, M. C. Iglesias-de la Cruz, F. Sanz-Rodríguez, J. Y. Chooi, P. Rodríguez-Sevilla, D. Choudhury, A. K. Kar, J. García Solé, L. Paterson, D. Jaque, "Heat in optical tweezers," Proc. SPIE 8810, Optical Trapping and Optical Micromanipulation X, 88102A (12 September 2013); doi: $10.1117 / 12.2027750$

SPIE Event: SPIE NanoScience + Engineering, 2013, San Diego, California, United States 


\title{
Heat in optical tweezers
}

\author{
B. del Rosal ${ }^{\mathrm{a}}$, P. Haro-González $z^{\mathrm{a}}$, W. T. Ramsay ${ }^{\mathrm{b}}$, L. M. Maestro ${ }^{\mathrm{a}}$, K. Santacruz-Gómez ${ }^{\mathrm{c}}$, M. C. \\ Iglesias-de la Cruz ${ }^{\mathrm{a}}$, F. Sanz-Rodríguez ${ }^{\mathrm{a}}$, J. Y. Chooi ${ }^{\mathrm{a}}$, P. Rodríguez-Sevilla ${ }^{\mathrm{a}}$, D. Choudhury ${ }^{\mathrm{b}}$. A. K. \\ Kar $^{\mathrm{b}}$, J. G. García-Soléa ${ }^{\mathrm{a}}$, L. Paterson ${ }^{\mathrm{b}}$ and D. Jaque*a \\ ${ }^{a}$ Fluorescence Imaging Group, Departamento de Física de Materiales, Facultad de Ciencias, \\ Universidad Autónoma de Madrid, Campus de Cantoblanco, Madrid 28049, Spain; ${ }^{\mathrm{b}} \mathrm{Scottish}$ \\ Universities Physics Alliance (SUPA), Institute of Biological Chemistry Biophysics and \\ Bioengineering, School of Engineering \& Physical Sciences, Heriot Watt University, Edinburgh \\ EH14 4AS, Scotland; ${ }^{\circ}$ Departamento de Física, Universidad de Sonora, 83000 Hermosillo, Sonora, \\ México
}

\begin{abstract}
Laser-induced thermal effects in optically trapped microspheres and single cells have been investigated by Luminescence Thermometry. Thermal spectroscopy has revealed a non-localized temperature distribution around the trap that extends over tens of microns, in agreement with previous theoretical models. Solvent absorption has been identified as the key parameter to determine laser-induced heating, which can be reduced by establishing a continuous fluid flow of the sample. Our experimental results of thermal loading at a variety of wavelengths reveal that an optimum trapping wavelength exists for biological applications close to $820 \mathrm{~nm}$. This has been corroborated by a simultaneous analysis of the spectral dependence of cellular heating and damage in human lymphocytes during optical trapping. Minimum intracellular heating, well below the cytotoxic level $\left(43^{\circ} \mathrm{C}\right)$, has been demonstrated to occur for optical trapping with 820 $\mathrm{nm}$ laser radiation, thus avoiding cell damage.
\end{abstract}

Keywords: Optical trapping, nanothermometry, quantum dots, fluorescence imaging, microspheres, lymphocytes

\section{INTRODUCTION}

Optical traps and tweezers have proven to be highly versatile tools that make it possible to manipulate single particles at the microscopic scale $e^{1-4}$. The applications of this non-contact manipulation technique cover a wide range of fields, from material studies ${ }^{5,6}$ to single cell manipulation ${ }^{7,8}$, allowing, for instance, the study of interactions between individual cells ${ }^{9}$. Optical trapping of micrometrical objects can be accomplished by focusing a laser beam through a high numerical aperture objective, thus achieving laser spots whose diameter is close to the diffraction limit.

Typically, the chosen wavelengths for trapping experiments lie in the NIR, where there are two spectral ranges (known as first and second biological windows) where the absorption of water and other components of biological specimens is at a minimum ${ }^{9}$. Laser powers required for optical trapping are on the order of a few hundred of miliwatts ${ }^{10}$. However, the tight focusing of the laser beam leads to laser power densities at the optical trap as high as $\mathrm{MW} / \mathrm{cm}^{2}$. Then, even when such appropriate wavelengths are used, at such high laser power any residual absorption will result in a non-negligible thermal loading at the trap position ${ }^{11-13}$.

Whereas sometimes laser induced-heating is desirable, such as in photothermal therapy of cancer tumors ${ }^{14}$, in trapping experiments temperature increments will be detrimental in most cases, leading to undesired changes in the properties of the studied systems. Moreover, when working with biological samples, even temperature increments below $5^{\circ} \mathrm{C}$ will cause alterations in the biochemistry and molecular machinery of cells ${ }^{15,16}$. If temperature reaches the cytotoxic level $\left(43^{\circ} \mathrm{C}\right)$, cell death will be triggered through apoptosis or necrosis ${ }^{17,18}$. In addition, the trapping dynamics itself can be altered when the temperature is increased, as other forces besides radiation pressure would become relevant ${ }^{11,}{ }^{12}$. Therefore, a full knowledge of the thermal loading in trapping processes is required to make a correct interpretation of the results of trapping experiments. Furthermore, when studying biological samples, a range of experimental parameters where no significant alterations or irreversible damage occur to the cells needs to be determined.

Optical Trapping and Optical Micromanipulation X, edited by Kishan Dholakia, Gabriel C. Spalding, Proc. of SPIE Vol. 8810, 88102A · C 2013 SPIE · CCC code: 0277-786X/13/\$18 · doi: 10.1117/12.2027750 
Due to the intrinsic interest of this subject, a great number of works that deal with laser-induced thermal loading in optical traps can be found in the literature. The great discrepancy between the reported heating rates (temperature increment per watt of laser power), which range between $15^{\circ} \mathrm{C} / \mathrm{W}$ and $70^{\circ} \mathrm{C} / \mathrm{W}^{11,12,19}$, can undoubtedly be attributed to the different experimental conditions and, more precisely, to the difference in laser wavelength. The model proposed by Mao et al. ${ }^{1913}$. states that the laser-induced temperature increment in an optical trap, $\Delta T_{\text {trap }}$, depends on the absorption coefficient of the fluid at the laser wavelength, $\alpha_{a b s}\left(\lambda_{\text {trap }}\right)$, on the laser power, $P_{l}$, the chamber thickness, $D$, the thermal diffusivity of the liquid medium, $K$, and the laser spot radius $w_{l}$ according to the following expression:

$$
\Delta T_{\text {trap }}=\frac{\alpha_{\text {abs }}\left(\lambda_{\text {trap }}\right) \cdot P_{l}}{2 \cdot \pi \cdot K} \cdot \ln \left[\frac{D}{w_{l}}\right]
$$

Taking into account that the laser spot radius is related to the numerical aperture, $N A$, of the objective through the following expression,

$$
w_{l}=\frac{0.6 \cdot \lambda_{\text {trap }}}{N A}
$$

the heating rate at an optical trap, $\beta_{\text {trap }}$, i. e. the temperature increment divided by the laser power, can be written as follows:

$$
\beta_{\text {trap }}=\frac{\Delta T_{\text {trap }}}{P_{l}}=\frac{\alpha_{\text {abs }}\left(\lambda_{\text {trap }}\right) \cdot P_{l}}{2 \cdot \pi \cdot K} \cdot \ln \left[\frac{D \cdot N A}{0.6 \cdot \lambda_{\text {trap }}}\right]
$$

From this expression, it can be clearly seen that laser wavelength is one of the main parameters determining the thermal loading in an optical trap. However, other factors besides thermal loading need to be taken into account when choosing an optimum wavelength for a given trapping experiment. For instance, photochemical processes that can occur in biological cells must be avoided in order not to affect cell viability ${ }^{20}{ }^{21}$. Therefore, for each trapping experiment an optimum wavelength which does not alter the sample while keeping the laser-induced heating at a minimum must be carefully determined.

In this work, luminescence thermometry (LTh), which takes advantage of temperature-induced changes in the emission signal of fluorescent probes ${ }^{22}$, has been used to study the thermal changes that occurred when optically trapping polystyrene microspheres. Both quantum dots and fluorescent dyes whose emission signals are strongly temperaturesensitive have been used as probes to measure the laser-induced heating in trapping experiments. These probes allow us to determine the thermal loading at the optical trap and its surroundings, where a heat-affected zone (HAZ) along which temperature follows an exponential decay with the distance to the laser focus, is expected to appear due to the thermal diffusivity of the medium ${ }^{19,23}$. This non-contact technique is especially suitable for temperature measurement in this kind of systems, as it makes it possible to determine the temperature without disturbing the experimental conditions. Besides, it presents good spatial and thermal resolution, allowing us to accurately determine the temperature distribution within an optical trap with sub-micrometrical resolution.

Taking advantage of this method, we have studied the laser-induced heating in optical traps, paying special attention to the effects of the wavelength and the absorption of the solvent. In addition, the possible effects of establishing a continuous flow of the sample throughout the trapping microchannel have also been analyzed.

\section{EXPERIMENTAL}

\subsection{Trapped microparticles}

Both polymer and silica microspheres, as well as human lymphocytes, were used to carry out the trapping experiments described in this work. The polymer microspheres were provided by Polysciences, Inc. They were uniform, monodisperse polystyrene microspheres (at. No. 17134, Polysciences, Eppleheim, Germany) $3 \mu \mathrm{m}$ in diameter. So as to avoid multi-particle trapping, they were dispersed in water at a concentration as low as $0.02 \mu \mathrm{g} / \mathrm{mL}$. 
For single-cell optical trapping experiments, Jurkat cells (clone E6.1) were used. Freshly cultured suspensions of these cells were maintained in RPMI 1640 medium supplemented with $10 \%$ fetal calf serum (FCS), $1 \%$ (v/v) penicillin/streptomycin $(10000 \mathrm{IU} / \mathrm{mL} / 10000 \mathrm{~g} / \mathrm{mL})$, and $2 \mathrm{mM} \mathrm{L}$-glutamine (complete medium) in an incubator with humidified $95 \%$ air, $5 \% \mathrm{CO} 2$ atmosphere at $37^{\circ} \mathrm{C}$. All reagents were purchased from Fisher Scientific (Massachusetts, USA). For experiments, an aliquot of $100 \mu \mathrm{L}$ of fresh, exponentially growing lymphocytes (cell density of $10^{6}$ cells $/ \mathrm{mL}$ ) were taken and subsequently diluted $1 / 5$ with complete medium. Immediately after, $50 \mu \mathrm{L}$ of this suspension were placed on a microscopy chamber (Ibidi, Germany).
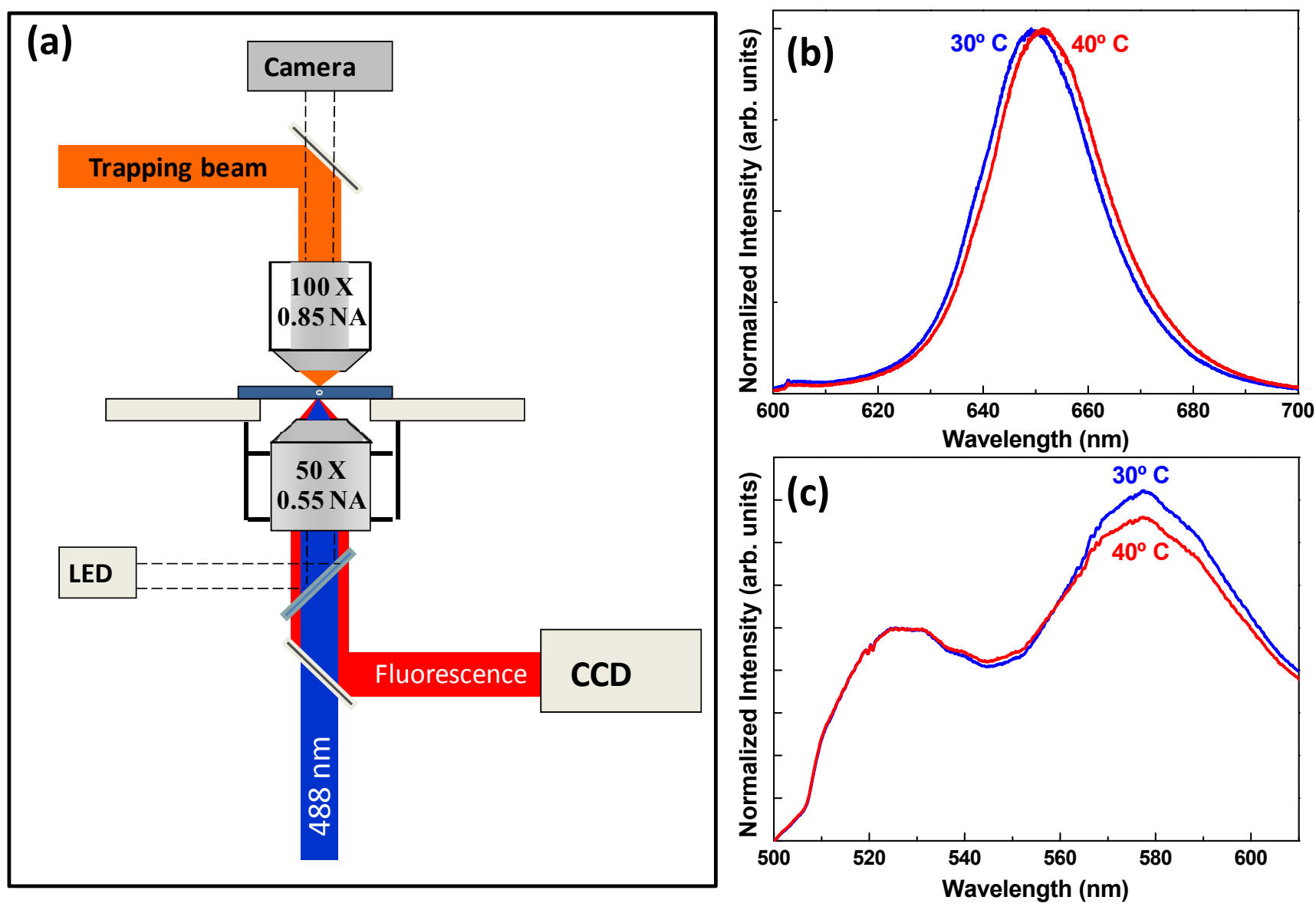

Figure 1. (a) Schematic diagram of the double-beam confocal microscopy used for thermal sensing and imaging of optical traps. (b) and (c) emission spectra of the fluorescent probes used in this work as temperature sensors, Cd-Se QDs (b) and solution containing RhB and Rh100 (c) at two different temperatures.

\subsection{Luminescent probes}

The two different luminescent probes were used as temperature sensors in this work were CdSe-QDs and a solution of Rhodamine $\mathrm{B}(\mathrm{RhB})$ and Rhodamine $110(\mathrm{Rh} 110)$, both of which have already been successfully used as temperature sensors in different systems ${ }^{24-26}$. The CdSe QDs were provided by Invitrogen, Inc. (Q21721MP) and had an average size of $14 \pm 3 \mathrm{~nm}$ in length and $6 \pm 0.6 \mathrm{~nm}$ in width. They present an emission band peaking at about $640 \mathrm{~nm}$, which suffers a noticeable redshift when the temperature increases, as can be seen in Figure 1(b). The luminescent dyes where provided by SigmaTech Inc., and their concentration in the solution was $0.25 \mathrm{mM}$ and $0.05 \mathrm{mM}$ for RhB and Rh110, respectively. A temperature increment will result in a quenching in the emission band of Rhodamine B, which peaks at about $525 \mathrm{~nm}$, whereas the emission of Rhodamine 110 (peaking at about $575 \mathrm{~nm}$ ) remains unchanged. The ratio between both emissions can be used to determine the temperature with an accuracy of $1^{\circ} \mathrm{C}$, and avoids any effect due to local changes in concentration or light intensity that could arise when only a temperature-sensitive dye were used. To perform 
temperature measurements, a $200 \mu \mathrm{m}$-deep microchannel (Ibidi, Inc.) was filled with either a solution containing CdSeQDs $\left(1 \cdot 10^{9} \mathrm{~cm}^{-3}\right)$ or the fluorescent dye solution previously described. To study the effects of the flow rate, a syringe pump (NE-1002X Programmable Microfluidics Pump) was used to establish a continuous flow of the dye solution along the microchannel.

\subsection{Temperature measurements}

In order to measure the temperature in the optical trap, two different experimental setups that allow for simultaneous microparticle trapping and excitation of the luminescent probes were used. First, a double-beam confocal microscope as the one depicted in Figure 1(a) was used to simultaneously create an optical trap and measure the laser-induced heating. Whereas the upper part of the microscope allows for multiple-wavelength trapping of micrometrical particles, the bottom section allows for excitation of the fluorescent probe and collection of the luminescence signal, thus making it possible to measure the temperature at the optical trap when the focal planes of both microscope objectives overlap. The $488 \mathrm{~nm}$ laser used to excite the luminescent probes was focused through a 50x objective $(\mathrm{NA}=0.55)$ which was also used to collect the luminescence signal. Meanwhile, to focus the trapping beam, a 100x objective with a numerical aperture (NA) of 0.8 was used. Five different trapping wavelengths were used throughout this work: $750 \mathrm{~nm}, 808 \mathrm{~nm}, 820 \mathrm{~nm}, 980 \mathrm{~nm}$ and $1090 \mathrm{~nm}$.

To obtain two-dimensional images in an easy way, a second experimental setup was used. While an identical trapping (top) section as the one depicted in Figure 1(a) was used, the bottom part of the previous setup was replaced with a commercial epi-fluorescence microscope. In this case, a high-voltage mercury lamp was used as an excitation source for the luminescent probes. After passing through an appropriate set of filters, the luminescence from each of the dyes was collected through a 20x microscope objective and recorded with a camera. We thus obtained two fluorescence images from which the temperature distribution could be reconstructed.

\section{RESULTS}

\subsection{Effect of the trapping wavelength}

The obtained heating rates $\left(\beta_{\text {trap }}\right)$ displayed in Figure 2 clearly reveal that wavelength is the critical parameter that determines the laser-induced heating in an optical trap. The experimentally obtained temperature increments as a function of the applied laser power at each wavelength, which can be seen in the inset of Figure 2, allowed us to calculate the heating rates represented in the figure. The linear dependence of the temperature increment on the power is in perfect agreement with expression (1), allowing us to conclude that the effect of any other process is negligible at these trapping wavelengths.

Moreover, the agreement between the experimentally determined heating rates for all five wavelengths and those predicted by to the model proposed by Mao et $\mathrm{al}^{13}$. is extremely good. It can be seen that the heating rate values follow the wavelength dependence of the absorption coefficient of water, which peaks at $980 \mathrm{~nm}$ in the range of wavelengths used throughout these works. At this trapping wavelength, the highest heating rate $\left(\beta_{\text {trap }}=99^{\circ} \mathrm{C} / \mathrm{W}\right)$ was observed.

\subsection{Effect of the solvent}

The fact that the experimentally obtained heating rates follow the wavelength dependence of the absorption coefficient of water points to the absorption of the solvent as the main factor determining the thermal loading in optical traps, in agreement to previous studies. In fact, a change of solvent results in a dramatic change in laser-induced temperature increment, as can be seen in Figure 3. The images shown represent the temperature distribution at the site of the optical trap and its surroundings using either water (Figure 3(b)) or heavy water $\left(\mathrm{D}_{2} \mathrm{O}\right)$ (Figure 3(c)) as a solvent for a trapping wavelength of $980 \mathrm{~nm}$. The absence of any significant heating for the case of heavy water can be perfectly explained taking into account its absorption spectrum, displayed along with that of water in Figure 3(a). 


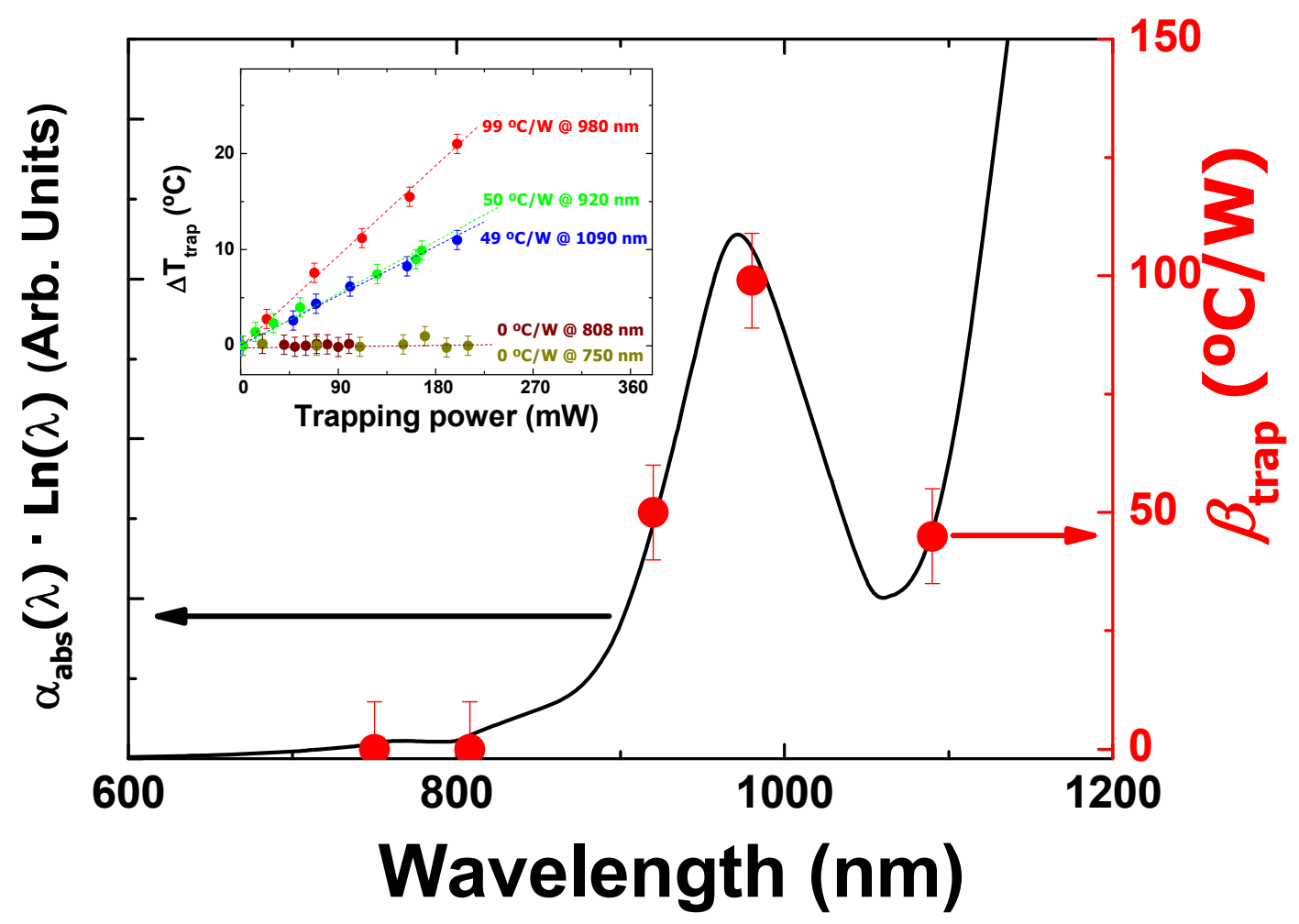

Figure 2. Heating rates at the five different wavelengths used in this work. Solid line indicates the wavelength dependence of the heating rate as predicted by the model given by Mao et. al. Insets shows the temperature increment data observed at different trapping powers for each wavelength, from which the heating rates have been calculated.
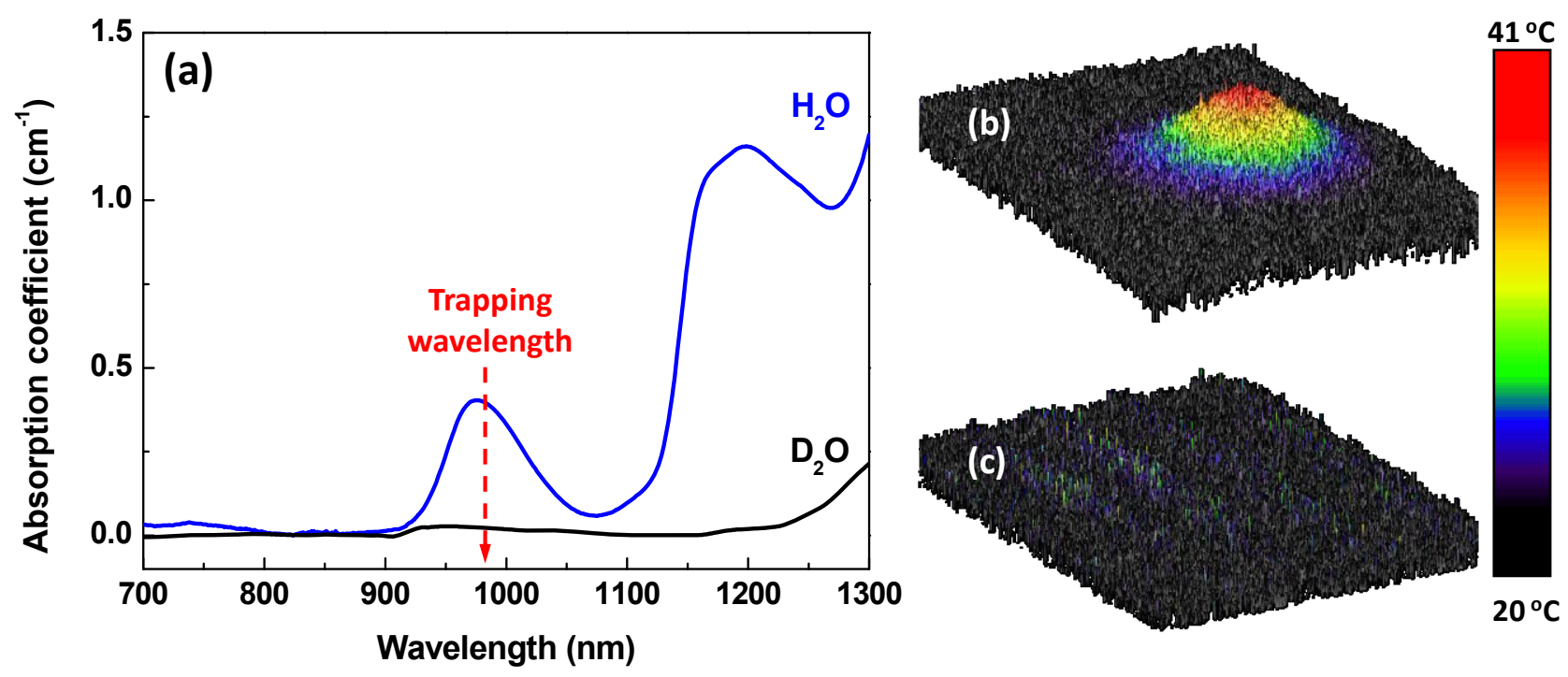

Figure 3. (a) Absorption spectra corresponding to water and heavy water. The trapping wavelength used to compare laserinduced heating in both solvents is marked with a dashed line. Note that the absorption coefficient of heavy water vanishes at this wavelength. (b) and (c) Temperature distribution maps of optical traps created by a $980 \mathrm{~nm}$ laser beam using water (b) and heavy water (c) as solvents, respectively in a $150 \mu \mathrm{m} \times 150 \mu \mathrm{m}$ area. The trapping power was set to $370 \mathrm{~mW}$ in both cases. 
While the absorption coefficient of water peaks at $980 \mathrm{~nm}$, absorption of heavy water is practically negligible at that wavelength. This difference between both absorption coefficients at that trapping wavelength accounts for the difference between the temperature distributions represented in Figures 3(b) and 3(c).

From the thermal images depicted in Figure 3, it can also be clearly seen that the laser-induced heating is not restricted to the optical trap, but instead it extends for tens of microns out of it. The existence of such a heat-affected zone (HAZ) was indeed expected due to the thermal diffusivity of the medium where the trapping occurs.

\subsection{Effect of the fluid flow}

Up to this moment, we have worked with static solutions containing fluorescent temperature-sensitive probes to analyze the effect of the wavelength and the solvent in the laser-induced heating. However, if the sample containing the microparticles to be trapped is pushed through the microchannel in a continuous flow-through manner, it is expected that the temperature increment will be significantly reduced with respect to the static case.

As can be seen in Figure 4, which represents the maximum temperature increment for different particle velocities in an optical trapping experiment at $980 \mathrm{~nm}$, thermal loading does indeed decrease as the flow rate (and thus, the speed of the particles) of the sample increases. As is displayed in figure 4, the temperature rise at the highest flow rate studied in this work, which still allows for microsphere trapping, is significantly lower (close to $30^{\circ} \mathrm{C}$ ) to that occurring when no flow is applied. Therefore, establishing a continuous fluid flow is a suitable way of minimizing the laser-induced heating when the required laser wavelength and power cause a significant temperature increment in the sample.

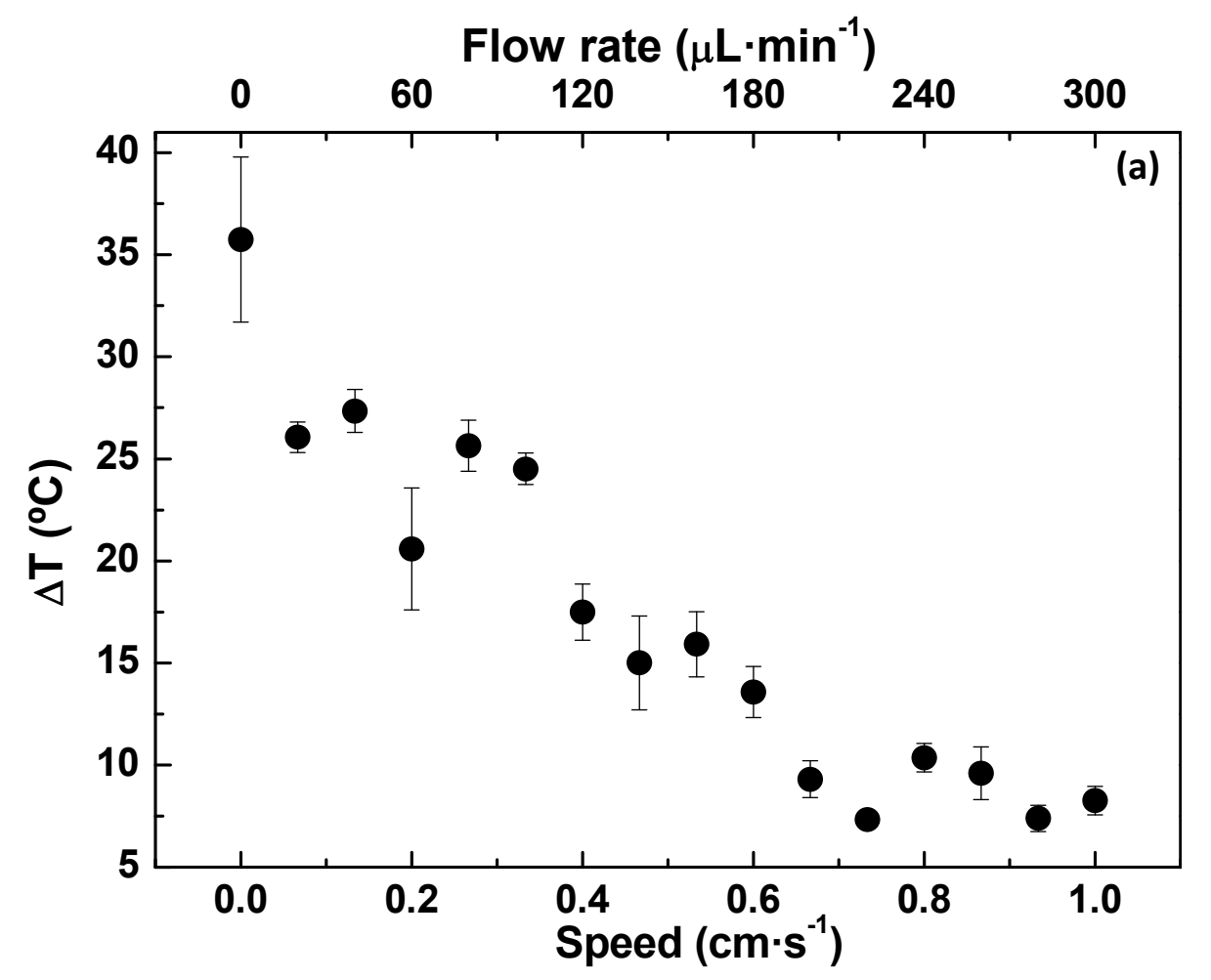

Figure 4. (a) Maximum temperature increment that occurs at an optical trap for different particle speeds at a trapping wavelength of $980 \mathrm{~nm}$. The laser power was set to $370 \mathrm{~mW}$ in all cases. The corresponding flow rate of the sample through the trapping channel is indicated in the top axis.

\subsection{Laser-induced heating effects on biological cells}

As we have seen throughout this work, choosing the appropriate trapping parameters is key to minimizing the laserinduced heating in the sample. This is particularly important when working with biological samples, as any temperature increase above the cytotoxic level $\left(43^{\circ} \mathrm{C}\right)$ will result in irreversible damage to cells and biochemical changes would occur even for lower temperature increments. Taking in to account that water is the primary component of the cell itself and the medium surrounding it, it seems logical to assume that the wavelength dependence of the photothermal damage 
caused to the cell will be the same of the laser-induced heating in an aqueous solution depicted in Figure 2, which is given by the absorption coefficient of water. Therefore, a minimum thermal loading is expected for an excitation wavelength of $820 \mathrm{~nm}$.

The state of the cell during a trapping experiment can be evaluated by monitoring it under the microscope in real time, studying the occurrence of any king of visible cell damage (blebbing, membrane rupture) ${ }^{27}$. Loss of circularity is, in the case of the human lymphocytes used in our experiments, a convenient way to analyze cell damage. As can be seen in Figure 5, the laser-induced damage caused to the lymphocyte is as, expected, highly dependent on the wavelength. The morphology of the trapped lymphocytes was compared to that of healthy untrapped lymphocytes for a laser power of 300 $\mathrm{mW}$ at two wavelengths $(820 \mathrm{~nm}$ and $980 \mathrm{~nm})$ between which laser-induced heating varies greatly.
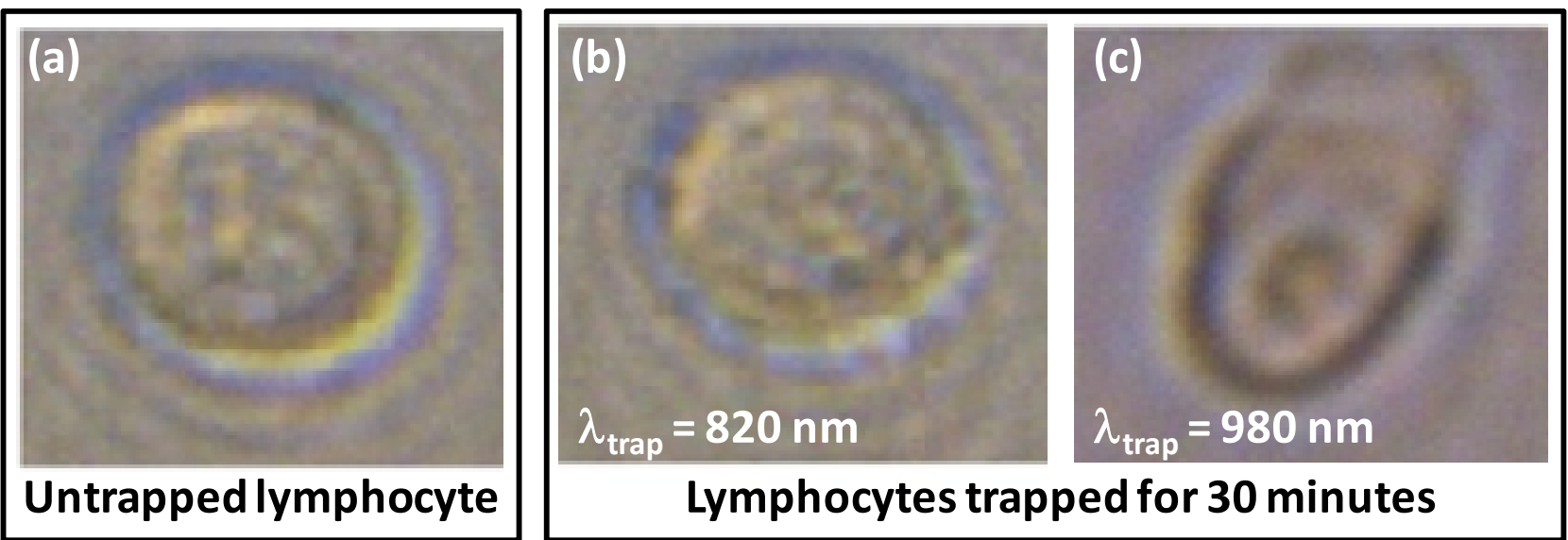

Figure 5. Optical transmission images of human lymphocytes (a)-before trapping and (b) and (c) after 30 minutes of trapping under laser wavelengths of $820 \mathrm{~nm}$ (b) and $980 \mathrm{~nm}$ (c), respectively. In both cases, the trapping power was set to $300 \mathrm{~mW}$.

For trapping times of 30 minutes, evident changes in the morphology of the lymphocyte trapped under the $980 \mathrm{~nm}$ laser beam have taken place and the aspect ratio has varied significantly. These morphological changes suggest that temperatures close to the cytotoxic level have been reached in the optical trap. In fact, results obtained by luminescence thermometry, not displayed here for the sake of brevity, reveal that at that laser power an intracellular temperature increment of about $20^{\circ} \mathrm{C}$ is expected. Taking into account that the experiments have been performed at room temperature $\left(22 \pm 1^{\circ} \mathrm{C}\right)$, laser-induced heating is effectively driving the cells very close to the cytotoxic level.

On the other hand, the shape of the lymphocyte trapped at a wavelength of $820 \mathrm{~nm}$ remains very similar to that of untrapped lymphocytes. These results reveal that, for $820 \mathrm{~nm}$, the laser-induced heating is not causing noticeable damage to the cell and besides, that no photochemical processes of significance are taking place. Therefore, $820 \mathrm{~nm}$ can be proposed as an optimum wavelength for biological applications of trapping as laser-induced damage will be kept at a minimum.

\section{CONCLUSIONS}

Throughout this work, thermal effects in optical traps have been analyzed by luminescence thermometry. Trapping wavelength has proven to be the critical parameter determining the temperature increase at a given laser power. The wavelength dependence of the laser-induced heating at an optical trap follows exactly that of the absorption coefficient of the solvent where the particles to be trapped are dispersed. Therefore, a maximum heating rate has been observed at $980 \mathrm{~nm}$ for aqueous solutions, whereas no significant heating is found at that wavelength for dispersions in $\mathrm{D}_{2} \mathrm{O}$. For biological samples, it has been determined that $820 \mathrm{~nm}$ constitutes an optimum trapping wavelength, simultaneously minimizing heating and photochemical damage of cells.

Moreover, the temperature distribution at the surroundings of optical traps has been studied, leading us to prove that the heat-affected area extends for tens of microns around the optical trap. Establishing a continuous flow of the sample has proven to affect the size and symmetry of this heat affected area but, more importantly, it has proven to substantially reduce the laser-induced heating at the optical trap. 


\section{REFERENCES}

[1] Ashkin, A., Dziedzic, J. M., and Yamane, T., "Optical trapping and manipulation of single cells using infraredlaser beams," Nature 330(6150), 769-771 (1987)

[2] Ashkin, A. and Dziedzic, J. M., "Optical trapping and manipulation of viruses and bacteria," Science 235(4795), 1517-1520 (1987)

[3] Stevenson, D. J., Gunn-Moore, F., and Dholakia, K., "Light forces the pace: optical manipulation for biophotonics," Journal of Biomedical Optics 15(4), (2010)

[4] Gu, M., et al., "Laser trapping and manipulation under focused evanescent wave illumination," Applied Physics Letters 84(21), 4236-4238 (2004)

[5] Wang, K., et al., "Trapping and rotating nanoparticles using a plasmonic nano-tweezer with an integrated heat sink," Nature Communications 2, (2011)

[6] Wright, W. H., Sonek, G. J., and Berns, M. W., "PARAMETRIC STUDY OF THE FORCES ON MICROSPHERES HELD BY OPTICAL TWEEZERS," Applied Optics 33(9), 1735-1748 (1994)

[7] Bankapur, A., et al., "Raman Tweezers Spectroscopy of Live, Single Red and White Blood Cells," Plos One 5(4), (2010)

[8] Snook, R. D., et al., "Raman tweezers and their application to the study of singly trapped eukaryotic cells," Integrative Biology 1(1), 43-52 (2009)

[9] Dholakia, K., Reece, P., and Gu, M., "Optical micromanipulation," Chemical Society Reviews 37(1), 42-55 (2008)

[10] Hormeno, S. and Arias-Gonzalez, J. R., "Exploring mechanochemical processes in the cell with optical tweezers," Biology of the Cell 98(12), 679-695 (2006)

[11] Liu, Y., et al., "Evidence for localized cell heating induced by infrared optical tweezers," Biophysical Journal 68(5), 2137-2144 (1995)

[12] Peterman, E. J. G., Gittes, F., and Schmidt, C. F., "Laser-induced heating in optical traps," Biophysical Journal 84(2), 1308-1316 (2003)

[13] Mao, H. B., et al., "Temperature control methods in a laser tweezers system," Biophysical Journal 89(2), 1308$1316(2005)$

[14] Moon, H. K., Lee, S. H., and Choi, H. C., "In Vivo Near-Infrared Mediated Tumor Destruction by Photothermal Effect of Carbon Nanotubes," ACS Nano 3(11), 3707-3713 (2009)

[15] Wetzel, F., et al., "Single cell viability and impact of heating by laser absorption," European Biophysics Journal with Biophysics Letters 40(9), 1109-1114 (2011)

[16] Foo, J. J., Liu, K. K., and Chan, V., "Thermal effect on a viscously deformed liposome in a laser trap," Annals of Biomedical Engineering 31(3), 354-362 (2003)

[17] Fiers, W., et al., "More than one way to die: apoptosis, necrosis and reactive oxygen damage," Oncogene 18(54), 7719-7730 (1999)

[18] Majno, G. and Joris, I., "Apoptosis, oncosis, and necrosis - an overview of cell-death," American Journal of Pathology 146(1), 3-15 (1995)

[19] Celliers, P. M. and Conia, J., "Measurement of localized heating in the focus of an optical trap," Applied Optics 39(19), 3396-3407 (2000)

[20] Gross, S. P., "Application of optical traps in vivo," Biophotonics, Pt B 361, 162-174 (2003)

[21] Liang, H., et al., "Wavelength dependence of cell cloning efficiency after optical trapping," Biophysical Journal 70(3), 1529-1533 (1996)

[22] Jaque, D. and Vetrone, F., "Luminescence nanothermometry," Nanoscale 4(15), 4301-4326 (2012)

[23] Krasnikov, I., Seteikin, A., and Bernhardt, I., "Thermal processes in red blood cells exposed to infrared laser tweezers (lambda=1064 nm)," Journal of Biophotonics 4(3), 206-212 (2011)

[24] Maestro, L. M., et al., "CdTe Quantum Dots as Nanothermometers: Towards Highly Sensitive Thermal Imaging," Small 7(13), 1774-1778 (2011)

[25] Choudhury, D., et al., "Quantum dot enabled thermal imaging of optofluidic devices," Lab on a Chip 12(13), 2414-2420 (2012)

[26] Sakakibara, J. and Adrian, R. J., "Whole field measurement of temperature in water using two-color laser induced fluorescence," Experiments in Fluids 26(1-2), 7-15 (1999)

[27] Liang, H., et al., "Giant cell formation in cells exposed to $740 \mathrm{~nm}$ and $760 \mathrm{~nm}$ optical traps," Lasers in Surgery and Medicine 21(2), 159-165 (1997) 